\title{
MicroRNA-29b/142-5p contribute to the pathogenesis of biliary atresia by regulating the IFN- $\gamma$ gene
}

\author{
Yifan Yang', Zhu Jin', Rui Dong', Chao Zheng', Yanlei Huang', Yijie Zheng'ㄹ, Zhen Shen', Gong Chen', \\ Xiaoying Luo ${ }^{3}$ and Shan Zheng ${ }^{1}$
}

\begin{abstract}
Biliary atresia is one of the most common liver disease in infancy. The cause and pathogenesis remain largely unknown. This study aimed to investigate the potential regulatory effect of miR-29b/142-5p on IFN- $\gamma$ gene methylation. miRNAs microarray was performed on four pairs of liver and blood specimens from biliary atresia and choledochal cysts. We found the overexpression of miR-142-5p and mRNA level of DNA methyltransferase (DNMT) 1, and miR-29b and DNMT3a/DNMT3b were significantly negatively correlated in biliary atresia livers. Meanwhile, the methylation of the LINE-1, ALU and SAT2 repetitive sequences and the IFN- $\gamma$ promoter was lower, but the expression of IFN- $\gamma$ was upregulated. After transfected with DNMTs siRNAs, downregulation of DNMTs exerted a significant hypomethylating effect on the repetitive sequences, which led to upregulation of IFN- $\gamma$ in Jurkat cells. The direct interactions between miR-29b and DNMT3a/3b, and miR-142-5p and DNMT1 were identified using luciferase reporter assays. By transfecting mimics of miR-29b/142-5p into Jurkat cells, we found overexpression of miR-29b/142-5p markedly suppressed expression of DNMTs. Furthermore, the methylation of repetitive sequences and the IFN- $\gamma$ promoter region were remarkably downregulated, and with elevated IFN- $\gamma$ expression. After transfecting the miRNA inhibitors, the levels of DNMTs and the methylation of the IFN- $\gamma$ gene promoter region was upregulated, while levels of IFN- $\gamma$ were markedly suppressed. Our study suggested that miRNA-29b/142-5p overexpression and targeted inhibition of DNMTs expression resulted in decreased overall gene methylation and overexpression of the methylation-sensitive IFN- $\gamma$ gene.
\end{abstract}

\section{Introduction}

Biliary atresia is one of the most common causes of obstructive jaundice and the most common liver disease in infancy. It is characterized by progressive destructive hepatobiliary inflammation and fibrous obstruction, intrahepatic biliary fibrosis atresia, and eventually cirrhosis ${ }^{1,2}$.

\footnotetext{
Correspondence: X-Y. Luo (Ixybio@gmail.com) or

Shan Zheng (szheng@shmu.edu.cn)

'Department of Pediatric Surgery, Children's Hospital of Fudan University, and Key Laboratory of Neonatal Disease, Ministry of Health, Shanghai, China

${ }^{2}$ Medical Scientific Liaison Asian Pacific, Abbott Diagnostics Division, Abbott Laboratories, Shanghai 200032, China

Full list of author information is available at the end of the article.

These authors contributed equally: Yifan Yang, Zhu Jin

Edited by I. Amelio
}

While the etiology remains unclear, this worldwide problem has plagued innumerable pediatric surgeons.

Some studies have shown that biliary atresia is a type of CD4+ helper T-lymphocyte 1 (Th1)-mediated autoimmune diseases $^{3-6}$. IFN- $\gamma$ plays a crucial role during this process. Activated CD4+ Th1 cells secrete a large amount of IFN- $\gamma$, which induces a strong immune response, eventually leading to intrahepatic and extra-hepatic bile duct injury ${ }^{5,6}$. The extra-hepatic biliary tract of Ifn- $\gamma$-/- mice remained open after rotavirus infection. However, Ifn- $\gamma-/$ - mice that were infected with virus and recombinant IFN- $\gamma$ showed symptoms of biliary atresia, similar to the wild-type mice injected with virus ${ }^{7}$. This suggests that IFN- $\gamma$ plays a key role in the

\section{(c) The Author(s) 2018}

(c) (i) Open Access This article is licensed under a Creative Commons Attribution 4.0 International License, which permits use, sharing, adaptation, distribution and reproduction in any medium or format, as long as you give appropriate credit to the original author(s) and the source, provide a link to the Creative Commons license, and indicate if changes were made. The images or other third party material in this article are included in the article's Creative Commons license, unless indicated otherwise in a credit line to the material. If material is not included in the article's Creative Commons license and your intended use is not permitted by statutory regulation or exceeds the permitted use, you will need to obtain permission directly from the copyright holder. To view a copy of this license, visit http://creativecommons.org/licenses/by/4.0/. 

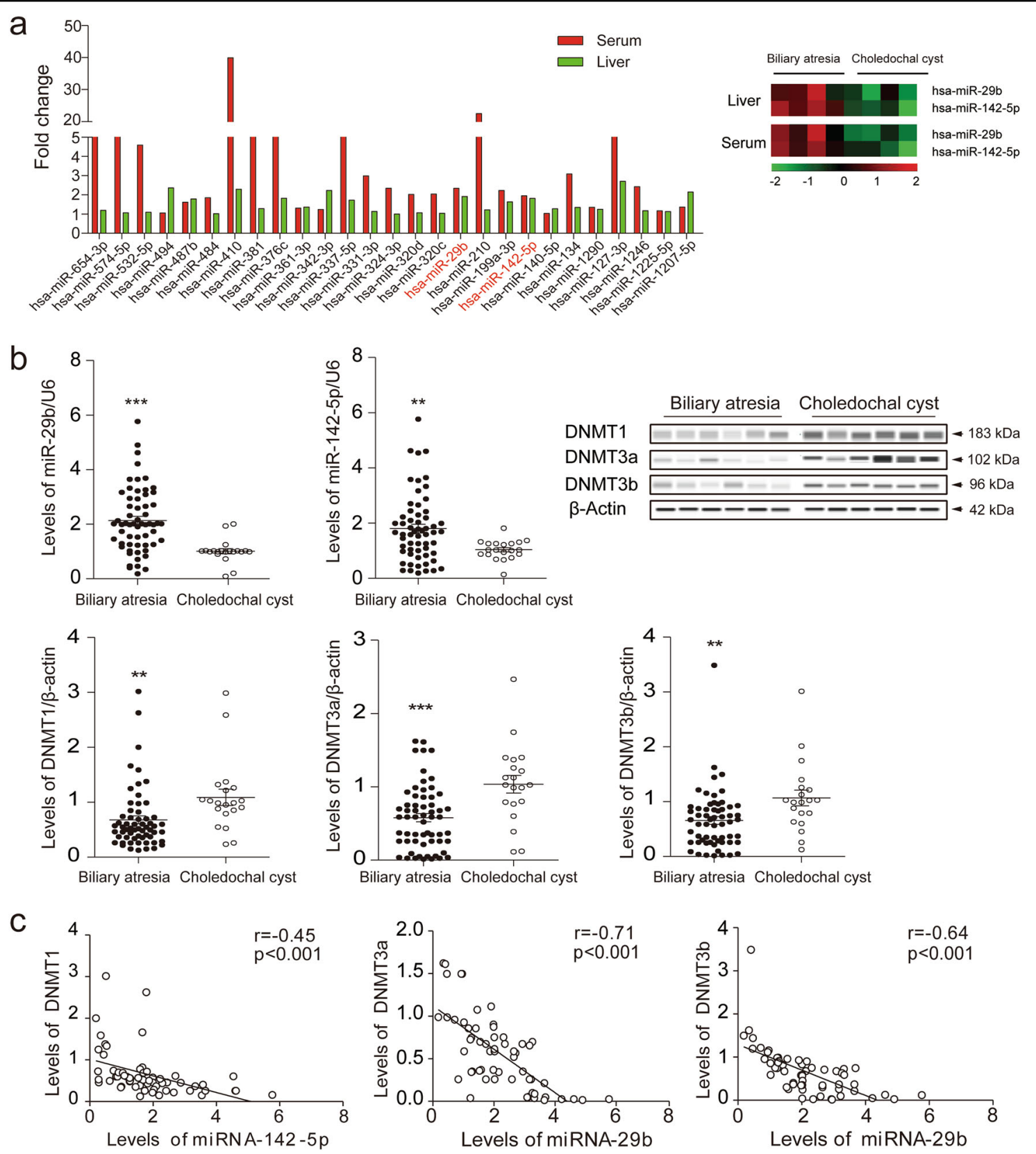

Fig. 1 Identification of miR-29b/142-5p overexpression and DNMTs downregulation in biliary atresia. a Twenty-seven differentially expressed miRNAs were both upregulated in liver and peripheral blood samples obtained from biliary atresia cases $(p<0.05$ with fold-change $>1.5)$ by miRNAs microarray; $\mathbf{b}$ Levels of miR-29b/142-5p in 60 biliary atresia and 16 choledochal cysts liver samples were detected using qPCR and normalized to U6 levels. Levels of DNMTs were detected by qPCR (normalized to $\beta$-actin) and western blotting. $\mathbf{c}$ The expression levels of miR-142-5p and DNMT1, and miR-29 and DNMT3a/DNMT3b mRNA were significantly negatively correlated $\left(p<0.001, r=-0.45,-0.71,-0.64\right.$, respectively). ${ }^{* *} p<0.01$,

${ }^{* * *} p<0.001$. Date represent mean values \pm SD

pathogenesis of biliary atresia in mice. The mechanism of IFN- $\gamma$ upregulation in the pathogenesis of biliary atresia and its biological function has consequently become the focus of the etiology in biliary atresia.

Recent studies have reported that epigenetics play an important role in the development of biliary atresia. Our previous results indicated that the genomic DNA of CD4+ T lymphocytes in the peripheral blood of children with biliary atresia was generally hypomethylated and that the expression of DNA methyltransferase (DNMT) 1 was significantly decreased. The hypomethylation of the IFN- $\gamma$ promoter region was negatively correlated with IFN $-\gamma$ 

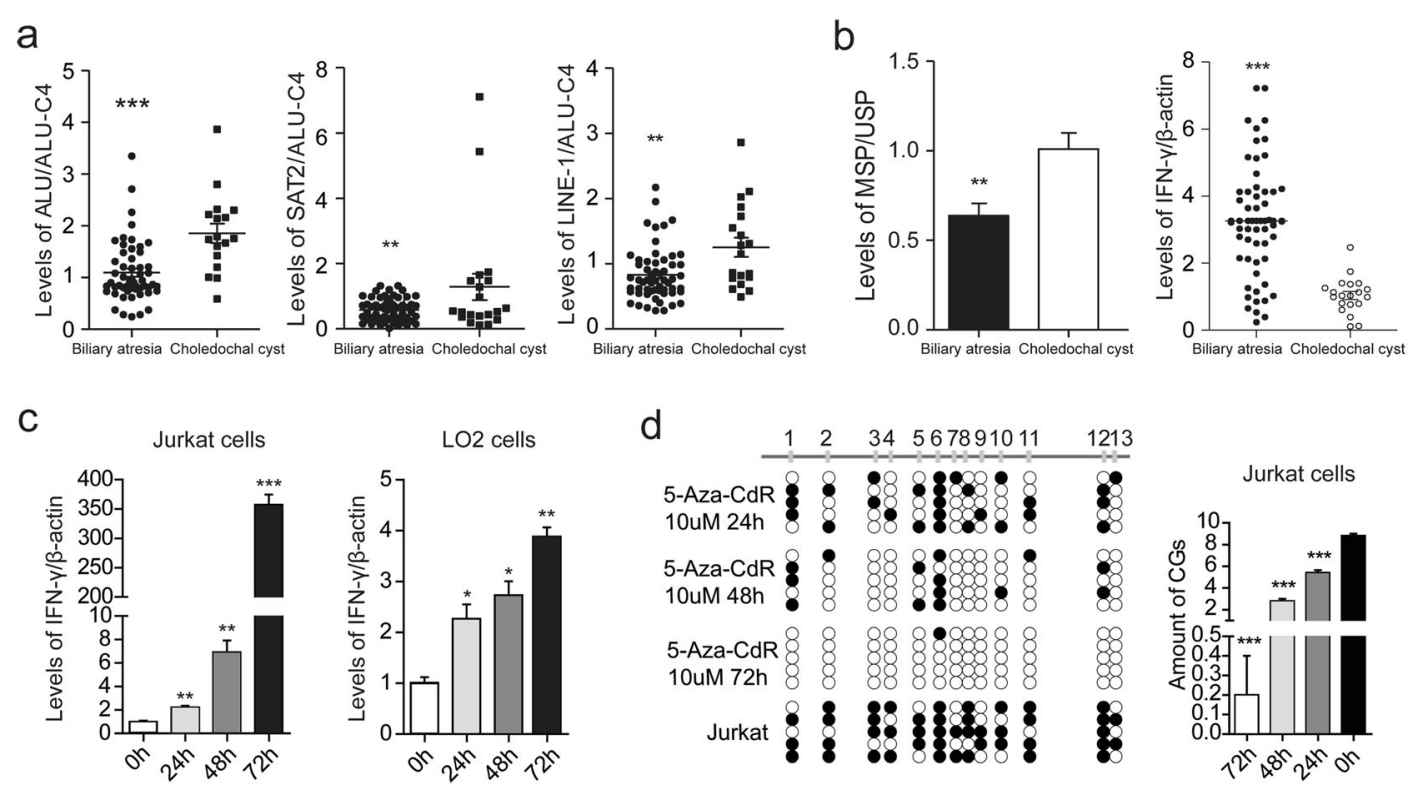

Fig. 2 Global genomic hypomethylation induced IFN- $\gamma$ upregulation in biliary atresia. a The quantitative methylation of the LINE-1, ALU, and SAT2 sequences were analyzed by methylation-specific PCR (MS-PCR) and normalized to ALU-C4 in biliary atresia liver samples. $\mathbf{b}$ The unmethylated (U) or methylated (M) IFN- $\gamma$ promoter sequence was carried out by MS-PCR in biliary atresia liver samples. Levels of IFN- - in 60 biliary atresia and 16 choledochal cysts liver samples were detected using qPCR and normalized to $\beta$-actin. c LO2 cells and Jurkat cells were treated with 5-aza-dC at different time points. Levels of IFN- $\gamma$ in both cells were detected using GPCR and normalized to $\beta$-actin. $\mathbf{d}$ The methylation of the IFN- $\gamma$ promoter were detected by bisulfite genomic sequencing at different time points in Jurkat cells. ${ }^{*} p<0.05,{ }^{* *} p<0.01,{ }^{* * *} p<0.001$. Date represent mean values \pm SD from three independent experiments

overexpression $^{8}$. Due to the large number of CpG islands in its promoter region serving as sites for methylation, the IFN- $\gamma$ gene is a methylation-sensitive gene ${ }^{9-11}$ Studies have shown that 5-aza-2'-deoxycytidine (5-aza-dC) inhibition of methylation could establish biliary atresia, and that bile duct injury is closely related to IFN- $\gamma$ expression in a zebrafish model ${ }^{12}$. These findings suggested that DNA hypomethylation is involved in the pathogenesis of biliary atresia, potentially through upregulation of IFN- $\gamma$. Additionally, our previous research findings indicated that there were many differentially expressed microRNAs (miRNAs) in children with biliary atresia ${ }^{13}$. These included miR-29b and miR-142-5p which might be related to methylation. Therefore, this study will further investigate the regulatory effect of miR-29b/142-5p on IFN- $\gamma$ gene methylation and its possible molecular mechanism.

\section{Results}

Identification of miR-29b and miR-142-5p overexpression in biliary atresia

To identify biliary atresia-specific miRNA profiles, miRNAs microarray detection was performed on four pairs of liver specimens and peripheral blood from biliary atresia and choledochal cysts cases. Fifty-two differentially expressed miRNAs were identified, among which 27 were upregulated (Fig. 1a), 3 were downregulated, and 22 were opposite in expression profiles of liver and peripheral blood samples obtained from biliary atresia cases $(p<0.05$ with fold-change $>1.5$ ). We further screen the miRNAs that may closely related to DNA methylation from these 30 differentially expressed miRNAs, and target prediction indicated that DNMT 3a and 3b were likely the target genes of miR29b while DNMT1 was the target gene for miR-142-5p.

For validation, expressions of miR-29b/142-5p and DNMTs in liver samples from 60 biliary atresia and 16 choledochal cysts were examined. We found that the expression of miR-29b and miR-142-5p were remarkably upregulated $(p<0.01)$, and that the messenger RNA (mRNA) and protein levels of DNMTs were downregulated $(p<0.05)$ in biliary atresia cases (Fig. 1b). The expression levels of miR-142-5p and DNMT1, and miR-29 and DNMT3a/DNMT3b mRNA were significantly negatively correlated ( $p<0.001$, Fig. 1 c).

\section{Global genomic hypomethylation induced IFN- $\gamma$ upregulation in biliary atresia}

The quantitative methylation analysis of the LINE-1, ALU, and SAT2 sequences showed that the methylation levels of these repetitive sequences were lower compared to controls $(p<0.01$, Fig. 2a). This suggests that genomic DNA was hypomethylated in all biliary atresia livers. Methylation-specific PCR was carried out for the unmethylated (U) or methylated (M) IFN- $\gamma$ promoter sequence. This showed that the IFN- $\gamma$ promoter was 
hypomethylated ( $p<0.01$, Fig. $2 \mathrm{~b})$, and that the expression of IFN- $\gamma$ was upregulated $(p<0.001$, Fig. $2 \mathrm{~b})$ in biliary atresia cases. To determine the effect of methylation on the IFN- $\gamma$ promoter and the associated expression of IFN$\gamma$, LO2 cells and Jurkat cells were treated with 5 -aza-dC at different time points. A significant increase in IFN- $\gamma$ expression levels were observed with increased times of treatment in both cell lines ( $p<0.05$, Fig. 2c). Notably, an increase of nearly 350-fold was recorded in Jurkat cells (Fig. 2c). We further investigated the methylation of the IFN- $\gamma$ promoter, and found that the ratio of $\mathrm{CpG}$ sites decreased with an increased time of treatment in Jurkat cells $(p<0.001$, Fig. $2 \mathrm{~d})$.

\section{Downregulation of DNMTs contributed to IFN- $\gamma$ promoter hypomethylation}

In this study, we found that the dramatically decreased mRNA levels of DNMTs were negatively correlated with the mRNA expression of IFN- $\gamma$ in biliary atresia cases $(p<0.001$, Fig. 3a). To confirm the suppressive role of DNMTs, DNMTs small-interfering RNA (siRNAs) were transfected into Jurkat cells to silence DNMTs expression. Downregulation of DNMTs exerted a significant hypomethylating effect on the ALU, LINE-1, and SAT2 repetitive sequences $(p<0.05$, Fig. $3 \mathrm{~b}, \mathrm{c})$, which induced hypomethylation of the IFN- $\gamma$ promoter and led to upregulation of IFN- $\gamma$ expression $(p<0.01$, Fig. 3d, e). Moreover, the levels of IFN- $\gamma$ mRNA were elevated 12fold in Jurkat cells transfected with three DNMT siRNAs, comparing the NC siRNA group (Fig. 3e).

\section{miR-29b/142-5p overexpression led to IFN- $\gamma$ upregulation by targeting DNMTs}

To confirm the target gene of miR-29b/142-5p, luciferase reporter assays were performed. Transfection with mimics of miR-29b and miR-142-5p could respectively inhibit the DNMTs wild-type vectors luciferase activity $(p<0.05)$, but failed to inhibit DNMTs mutant vectors luciferase activity in Jurkat cells (Supplementary Fig. 1). This indicated a direct interaction between miR-29b and DNMT3a/3b, and miR-142-5p, and DNMT1.

By transfecting mimics of miR-29b/142-5p into Jurkat cells, we evaluated the biologic role of miR-29b/142-5p. Overexpression of miR-29b/142-5p markedly suppressed expression of DNMT1, DNMT3a, and DNMT3b compared to the negative control. Furthermore, the methylation of LINE-1, ALU, and SAT2 sequences (Supplementary Fig. 2), and the IFN- $\gamma$ promoter region were remarkably downregulated, which was associated with elevated IFN- $\gamma$ expression (Fig. 4a, b, c). After transfecting the miRNA inhibitors, the levels of DNMTs were significantly elevated $(p<0.01)$, and the methylation of the IFN- $\gamma$ gene promoter region was upregulated, while IFN- $\gamma$ expression levels were markedly suppressed in Jurkat cell (Fig. 4d, e, f).
To further verify the effect of miR-29b/142-5p on IFN- $\gamma$ expression, the two miRNA mimics or inhibitors were transfected into Jurkat cells at the same time. The levels of IFN- $\gamma$ mRNA were elevated in miR-29b/142-5p co-mimics group than other three groups (Fig. 4c), while IFN- $\gamma$ mRNA were decreased in miRNA co-inhibitors group than other three groups (Fig. 4f). In addition, the miR-29b/142-5p coinhibitors and 5-aza-dC were transfected into Jurkat cells for $48 \mathrm{~h}$. We found the expression of IFN- $\gamma$ were higher in miR-29b/142-5p co-inhibitors with 5 -aza-dC group than the miRNA co-inhibitors group (Fig. 5a). However, there was no significant difference compared with miRNA NC inhibitor group (Fig. 5a). These results indicate that miR29b/142-5p could regulate the expression of IFN- $\gamma$ by targeting DNMTs (Fig. 5b).

\section{Discussion}

Numerous studies have shown that miRNAs, which are mainly involved in cellular regulation at post-transcriptional levels, are associated with a variety of biological processes, which include fetal development, organ formation, and cell proliferation and apoptosis ${ }^{14-16}$. Friedman et al. found that miR-29 was involved in the regulation of biliary atresia in a mouse model through its targeting of the Igf 1 gene $^{17}$. In 2013, Japanese scholars reported that miR-29a/b1 could downregulate the levels of DNA methylation by targeting DNMT3, and found that miR-29b was closely associated with type I collagen synthesis during cirrhosis ${ }^{18,}{ }^{19}$. Sonkoly et $\mathrm{all}^{20}$ found that miRNA-142-5p was significantly increased in some autoimmune diseases. In the present study, miRNA microarrays showed that miR-29b and miR$142-5 p$ overexpression was present in the liver and peripheral blood samples of biliary atresia patients. Closely related to DNA methylation, luciferase assays confirmed that these miRNAs target DNMT genes $\left(D N M T 1^{21}\right.$, DNMT3a, and DNMT3b22).

DNA methylation is currently one of the most studied and important forms of epigenetic modification ${ }^{23,} 24$. DNA methyltransferase can be inhibited or destroyed by a variety of causes leading to genome-wide hypomethylation status ${ }^{25}$. DNA hypomethylation exists in numerous autoimmune diseases, which can promote the overexpression of methylation-sensitive genes, such as: PRF1, $I F N-\gamma, C D 70^{26}$. It can also lead to over-activation of $\mathrm{T}$ cells caused by structural changes to the chromosomes associated with $\mathrm{T}$ cells ${ }^{27}$. Activated $\mathrm{CD} 4+\mathrm{Th} 1$ cells secrete large amounts of IFN- $\gamma$ and induce a strong immune response. Biliary atresia is also commonly recognized as an autoimmune disease, which induces the secretion of large quantities of IFN- $\gamma$. This eventually leads to intrahepatic bile duct injury ${ }^{6}$. Our study showed that DNMT1, DNMT3a, and DNMT3b expression is significantly decreased, while the expression level of IFN- $\gamma$ is increased in liver samples of biliary atresia cases. 


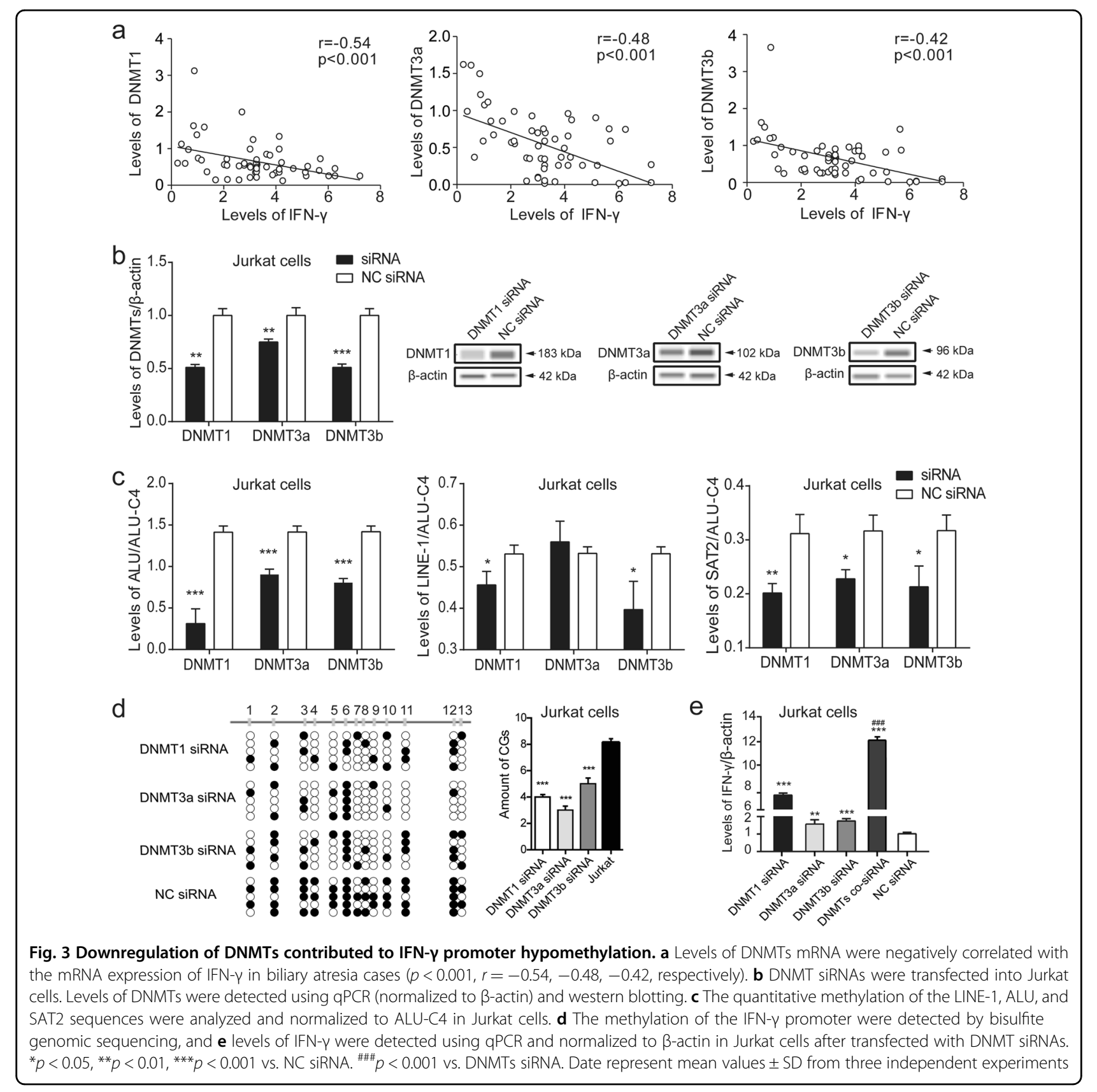

A large number of repetitive sequences account for about $45 \%$ of the entire sequence of the human genome. Of these, the ALU sequence accounts for about $10 \%$ of the entire genome and consists of 1 million copies. The LINE1 sequence is also a very important repeat sequence in the genome. The methylation status of these repeats is closely related to the overall methylation status of the genome and can, therefore, be used to measure the global DNA methylation levels ${ }^{28}$. By measuring the methylation status of the ALU, LINE-1, and SAT2 repetitive sequences ${ }^{29}$, we found that the methylation levels were significantly lower in biliary atresia cases than those in controls group. It could consequently be stated that DNA methylation levels were reduced globally in samples from biliary atresia patients. In addition, we found that the methylation levels of LINE-1 did not change, but that ALU and SAT2 were significantly altered after transfection with DNMT3a siRNA, mimics of miR-29b, and an inhibitor of miR-142$5 \mathrm{p}$. According to the research by Daniel et al. ${ }^{28}$, the correlation coefficient between global methylation level and ALU combined with SAT2 is $0.85, p<0.0001$, which is to say that an elevation or decrease in both ALU and SAT2 may indicate a corresponding increase or decrease in global methylation levels. 

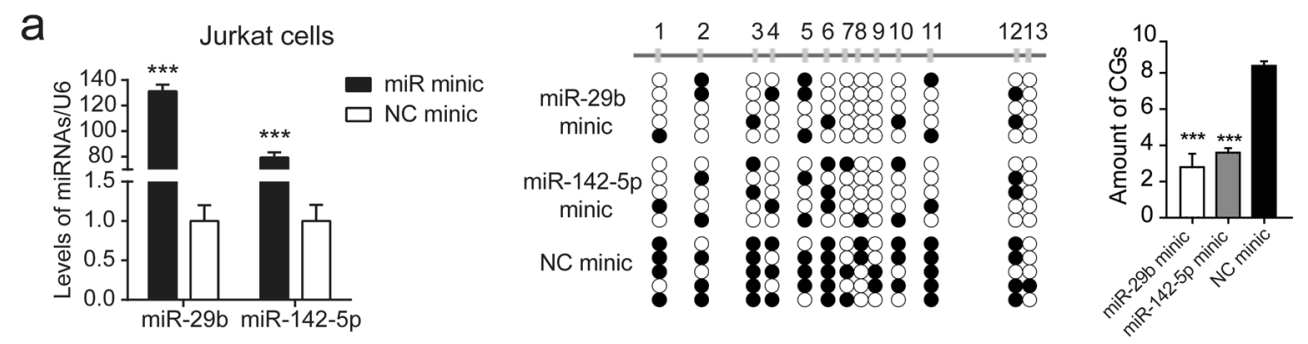

$\mathrm{b}$
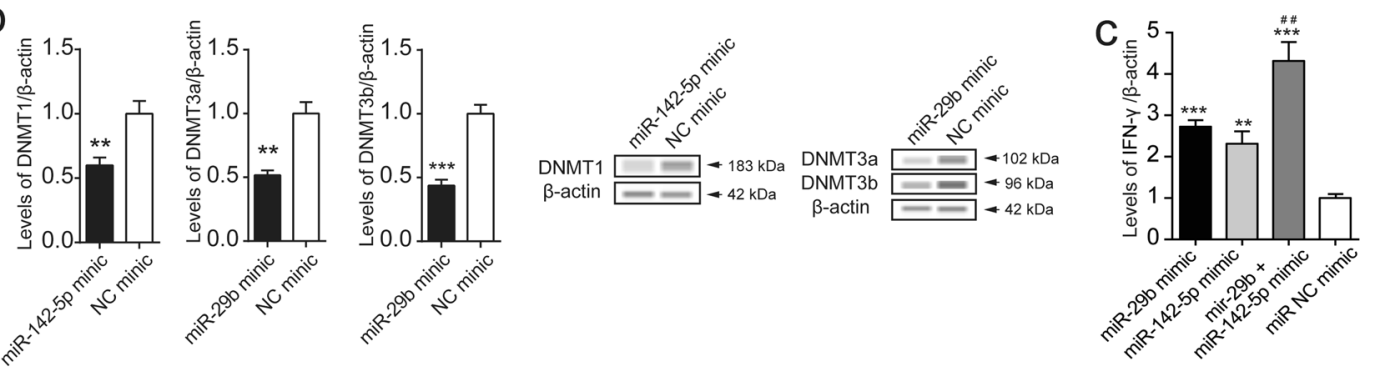

d
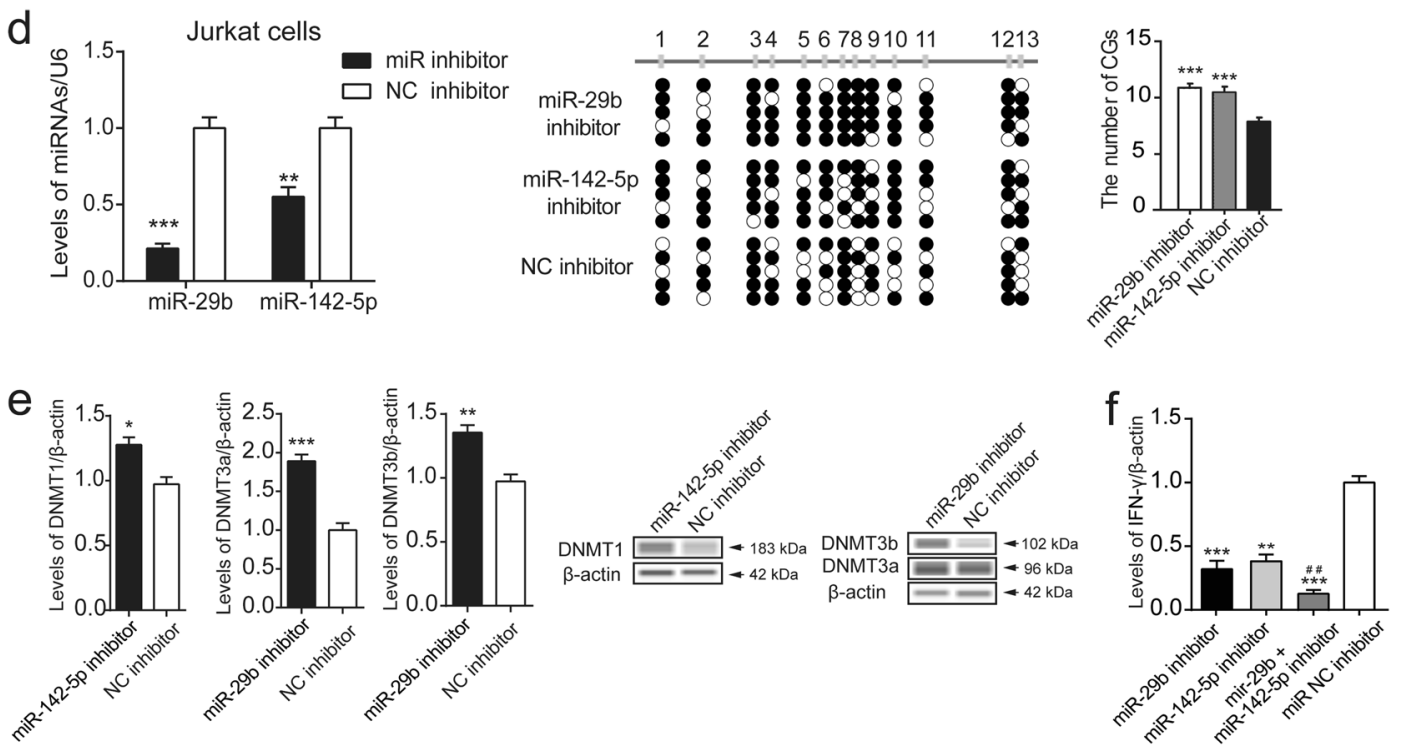

Fig. 4 Overexpression of miR-29b/142-5p led to IFN- $\gamma$ upregulation by targeting DNMTs. a Mimics of miR-29b/142-5p were transfected into Jurkat cells. Levels of miR-29b/142-5p were detected using qPCR and normalized to U6 levels. The methylation of the IFN- $\gamma$ promoter were detected by bisulfite genomic sequencing in Jurkat cells. $\mathbf{b}$ Levels of DNMTs were detected by qPCR (normalized to $\beta$-actin) and western blotting. $\mathbf{c}$ Levels of IFN- $\gamma$ were detected by qPCR and normalized to $\beta$-actin. d MiR-29b/142-5p inhibitors were transfected into Jurkat cells. Levels of miR-29b/142-5p were detected using qPCR and normalized to U6 levels. The methylation of the IFN- $\gamma$ promoter were detected. e Levels of DNMTs were detected by qPCR (normalized to $\beta$-actin) and western blotting. $\mathbf{f}$ Levels of IFN- $\gamma$ were detected by $q P C R$ and normalized to $\beta$-actin. ${ }^{*} p<0.05$, ** $p<0.01$,

${ }^{* * *} p<0.001$ vs. miRNA NC mimic or inhibitor. ${ }^{\# \#} p<0.001$ vs. miRNAs mimic or inhibitor. Date represent mean values \pm SD from three independent experiments

Using 5-aza-dC to inhibit genomic methylation of Jurkat and LO2 cells ${ }^{30}$, we found that IFN- $\gamma$ secreted from Jurkat cells increased by at nearly 350-fold, and confirmed that IFN- $\gamma$ was mainly produced by $\mathrm{T}$ lymphocytes ${ }^{31}$. Therefore, Jurkat cells, which resemble human leukemia $\mathrm{T}$ cells, were transfected with DNMTs siRNA. The results showed that the global methylation of genomic DNA was reduced, and the methylation of IFN $-\gamma$ promoter was decreased. This led to the overexpression of IFN- $\gamma$. To further investigate the effect of miR-29b/142-5p overexpression on IFN- $\gamma$, Jurkat cells were transfected with a mimic of miR-29b/142-5p. The expression of DNMT mRNA and proteins was decreased, while the methylation level of the IFN- $\gamma$ promoter was decreased. This resulted in the abundant expression of IFN$\gamma$. This was consistent with the hypothesis that biliary atresia pathogenesis is associated with increased miR-29b/ 
a

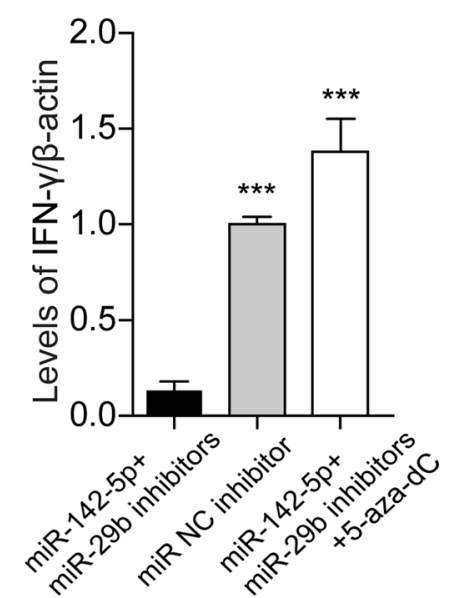

b

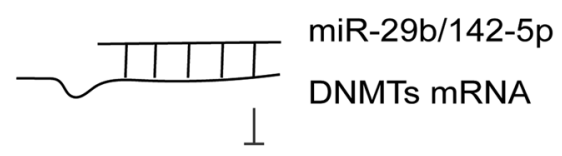

DNMTs $(1,3 a, 3 b)$

Global genomic methylation

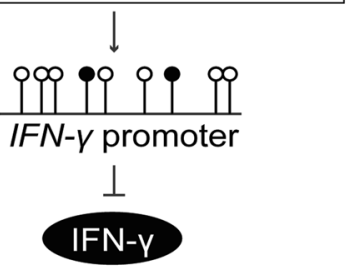

Fig. 5 Effect of miR-29b/142-5p on IFN- $\gamma$ expression. a The inhibitors of miR-29b/142-5p and $10 \mu \mathrm{M}$ 5-aza-dC were transfected into Jurkat cells for $48 \mathrm{~h}$. Levels of IFN- $\gamma$ were detected by $\mathrm{QPCR}$ and normalized to $\beta$-actin. b Diagram summarizing the findings. ${ }^{* * *} p<0.001$ vs. miRNA co-inhibitors. Date represent mean values \pm SD from three independent experiments

142-5p expression and T-lymphocyte activation. This is further hypothesized to lead to elevated concentrations of IFN- $\gamma$, which induces or aggravates biliary atresia. Similarly, we found that miRNA inhibitors significantly increased DNMTs expression in Jurkat cells, which resulted in decreased expression of IFN- $\gamma$.

In summary, this study found that miRNA-29b/142-5p overexpression and targeted inhibition of DNMTs expression resulted in decreased overall gene methylation and overexpression of the methylation-sensitive IFN- $\gamma$ gene. This may induce or aggravate the occurrence of biliary atresia. This study provides some evidence that further enhances our understanding of biliary atresia from the perspective of epigenetics while simultaneously exploring the pathogenesis of biliary atresia.

\section{Materials and methods}

\section{Patient samples and cells}

Sixty patients with type III biliary atresia and 20 patients with choledochal cysts who were treated at Children's Hospital of Fudan University from October 2013 to July 2014 were enrolled (Table 1). All patients were diagnosed with biliary atresia by exploratory laparotomy with operative cholangiography, and underwent successful Kasai portoenterostomy. Choledochal cysts patients without jaundice have normal liver function served as controls. This study was approved by the Human Ethics Boards at Children's Hospital of Fudan University. Written informed consent was obtained from the legal guardians of all subjects before starting study procedures. For RNA and protein extraction, tissue and blood samples were immediately snap-frozen and stored at $-80^{\circ} \mathrm{C}$.
Table 1 Characteristics of patients with biliary atresia (BA) or choledochal cysts (CCs)

\begin{tabular}{llll} 
& BA & CCs & $\boldsymbol{p}$-value \\
\hline Age (months) & $2.1 \pm 0.6$ & $18.5 \pm 3.2$ & $>0.05$ \\
Male & 35 & 11 & $\mathrm{~N} / \mathrm{A}$ \\
Female & 25 & 9 & $\mathrm{~N} / \mathrm{A}$ \\
Type & III $^{\mathrm{a}}$ & $\mathrm{I}^{\mathrm{b}}$ & $\mathrm{N} / \mathrm{A}$ \\
ALP $(\mathrm{IU} / \mathrm{L})$ & $625.5 \pm 28.7$ & $77.6 \pm 12.4$ & $<0.05$ \\
ALT $($ IU/L) & $122.6 \pm 20.2$ & $12.6 \pm 5.6$ & $<0.05$ \\
AST $($ IU/L) & $176.4 \pm 22.4$ & $18.3 \pm 7.2$ & $<0.05$ \\
DBIL (umol/L) & $121.1 \pm 6.2$ & $3.2 \pm 1.2$ & $<0.05$ \\
TBIL (umol/L) & $145.1 \pm 12.2$ & $7.8 \pm 2.3$ & $<0.05$ \\
GGT (IU/L) & $686.8 \pm 86.4$ & $36.4 \pm 13.6$ & $<0.05$ \\
TBA (umol/L) & $126.2 \pm 8.2$ & $7.5 \pm 2.2$ & $<0.05$ \\
\hline Type & & & \\
\end{tabular}

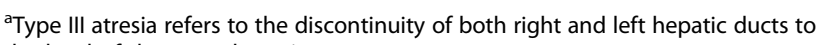
the level of the porta hepatis

${ }^{\mathrm{b}}$ Type $\mathrm{I}$ is the most common (80-90\%) involving saccular or fusiform dilatation of a portion or entire common bile duct with normal intrahepatic duct. CCs patients without jaudice have normal liver function as controls

The Jurkat human leukemia T-cell line and LO2 human normal liver cell line were obtained from the Institute of Biochemistry and Cell Biology at the Chinese Academy of Science (TCHu 69, Shanghai, China). All the cells were cultured in RPMI-1640 supplemented with 10\% FBS, 100 $\mathrm{IU} / \mathrm{mL}$ penicillin, and $100 \mathrm{mg} / \mathrm{mL}$ streptomycin sulfates. Treated daily with 5-aza-dC (Sigma-Aldrich, Steinheim, Germany) at a final concentration of $10 \mu \mathrm{M}$ for 24,48 , and $78 \mathrm{~h}$, Jurkat and LO2 cells were seeded at a density of $1 \times$ 106 cells $/ \mathrm{mL}$ and $5 \times 105$ cells $/ \mathrm{mL}$, respectively. 


\section{miRNA expression profiling}

Total miRNAs were extracted from tissue and blood samples according to the manufacturer's instructions by using mirVana miRNA isolation kit (Applied Biosystems, Foster City, CA, USA). As described in our previous study ${ }^{32}$, expression of miRNA was determined using miRNA microarrays (Agilent Technologies Inc., Santa Clara, CA, USA). Then, differentially expressed miRNAs were identified using the paired $t$-test with the cutoff criteria of $p<0.05$ and |fold change $\mid>1.5$. In order to ensure the screened differentially expressed miRNAs were accurately identified, hierarchical clustering analysis of samples was employed using heatmap. 2 function of the gplots package in $\mathrm{R}^{25}$ based on the expression values.

RNA extraction and quantitative reverse transcription PCR

Total RNA was extracted from cells or tissue with TRIzol reagent (Invitrogen, Carlsbad, CA, USA). Total miRNA quantification was achieved by quantitative reverse transcription PCR (qRT-PCR) using the miScript SYBR Green PCR Kit (Qiagen, Hilden, Germany) and the ABI 7500 Real-Time PCR System (Applied Biosystems). The U6 small nuclear RNA served as a reference. DNMT1, DNMT3a, DNMT3b, and IFN- $\gamma$ mRNA levels were determined using SYBR Green method, with $\beta$-actin as an internal reference. Primer sequences are shown in Supplementary Table 1 . The qRT-PCR results were analyzed with the $2^{-\Delta \Delta C t}$ method.

\section{Western blot analysis}

Snap-frozen liver samples and cells were homogenized in RIPA lysis buffer (Thermo Fisher Scientific, Hudson, $\mathrm{NH}$, USA). Equal amounts of protein were subjected to sodium dodecyl sulfate polyacrylamide gel electrophoresis followed with wet transfer to nitrocellulose membranes. The membranes were blocked with $5 \%$ skim milk, and incubated with the primary antibodies for the DNMT1, DNMT3a, DNMT3b, IFN- $\gamma$ and $\beta$-actin proteins (Abcam, Cambridge, MA, USA). After being incubated with a corresponding horseradish peroxidase-coupled secondary antibody (Beyotime, Shanghai, China), the membrane was allowed to react with the Lumi-Light ECL substrate (Thermo Fisher Scientific). ImageJ was then used to analyze the western blot result.

\section{DNA extraction and methylation analysis}

Genomic DNA was isolated from liver tissues, Jurkat and LO2 cells using the DNA Isolation kit (Tiangen, Beijing, China) according to the manufacturer's protocol. Determination of bisulfite conversion was performed using the EpiTect Bisulfite Kit (Qiagen). LINE-1, ALUM2, and SAT2-M1 methylation levels were measured through Methylation-specific PCR analysis as described in previous study ${ }^{33}$. Primer sequences for each repetitive sequence is as follows: LINE-1-M1 (forward: 5'GGACGTATTTGGAAAATCGGG-3'; reverse: $5^{\prime}$ AATCTCGCGATACGCCGTT-3'), ALU-M2 (forward: 5'-GCGCGGTGGTTTACGTTT-3'; reverse: 5'-AACCGAACTAATCTCGAACTCCTAAC-3'), and SAT2-M1 (forward: $\quad 5^{\prime}$-TCGAATGGAATTAATATTTAACGGA AAA-3'; reverse: 5'-CCATTCGAATCCATTCGATAAT TCT-3'). The bisulfite sequencing PCR reaction system was composed of $10 \times$ PCR buffer, $5.25 \mu \mathrm{M}$ dNTP Mix, $0.5 \mu \mathrm{M}$ of each primer, $0.75 \mathrm{U}$ hot start DNA polymerase, and 20 ng bisulfite modified DNA. Products from bisulfate PCR reactions were analyzed on $2 \%$ agarose gels and purified using QIAquick Gel Extraction Kit (Qiagen). Each purified product was cloned into a pMD 19-T Vector (Takara, Tokyo, Japan) and then transfected into Escherichia coli DH5 $\alpha$ competent cells (Vazyme Biotech Co., Piscataway, NJ, USA). Five clones from each sample were sequenced (Shinegene Molecular Biotechnology Co., Shanghai, China).

\section{Luciferase reporter assay}

DNMT (DNMT1, DNMT3a, and DNMT3b) mRNA 3'-UTR fragments containing the putative miR-29b/ 142-5p-binding sequence were amplified through PCR and cloned downstream of the luciferase reporter gene between the XbaI and EcoRI cutting sites of the pGL3control vector. The primers used for the DNMT-3'UTR clones are listed in Supplementary Table 2. Jurkat cells were co-transfected with pGL3-DNMT-3'-UTR or pGL3-DNMT-3'-UTR-mut, with cell extracts prepared $24 \mathrm{~h}$ after transfection. Luciferase activity was measured with the Dual-Luciferase Reporter Assay system (Promega, Madison, WI, USA) according to the manufacturers' protocol.

\section{miRNA target prediction}

At least two databases of the following five usual prediction databases: TargetScan (http://www.targetscan.org) and MiRanda (http://www.microrna.org/microrna/home. do), PicTar (http://pictar.mdc-berlin.de/), MirTarget2 from miRDB (http://mirdb.org/miRDB/ download.html) and PITA (http://genie.weizmann.ac.il/pubs/mir07/ mir07_prediction.html) were used to predict miRNA targets and conserved sites bound by the seed region of miR-29b and miR-142-5p in silico.

\section{Transfection}

Jurkat cells were transfected with either $20 \mathrm{nmol} / \mathrm{L}$ DNMT siRNAs or negative control (NC) siRNA (Biotend, Shanghai, China), $20 \mathrm{nmol} / \mathrm{L}$ of one of the following: a mimic of miR-29b/142-5p, or an inhibitor of miR-29b/ 142-5p, or a mimic/inhibitor NC, and CY3 dye as positive control for $48-72 \mathrm{~h}$. These were achieved using 
Lipofectamine 2000 Transfection Reagent (Thermo Fisher Scientific) according to the manufacturer's protocol. The transfection efficiency of DNMT siRNAs and miRNA mimic or inhibitor were $>90 \%$.

\section{Statistical analysis}

Data were presented as the means \pm standard deviation of at least three experiments. Statistical analysis was performed using SPSS 19.0 (SPSS, Chicago, IL, USA). An unpaired $t$ test was applied for statistical comparison between biliary atresia and choledochal cysts groups, DNMT siRNAs and negative control groups, and miRNA mimic or inhibitor and negative control groups. Analysis of variance was used to analyze different time points of 5-aza-dC groups. Pearson's correlation coefficient was used for correlation analysis between miRNAs and DNMTs expression. $p$-value $<0.05$ was considered statistically significant.

\section{Acknowledgements}

This study received financial support from Shanghai Hospital Development Center (SHDC12014106), Shanghai Key Disciplines (no.2017ZZ02022), National Natural Science Foundation of China (no. 81370472, no. 81770519, no. 81771633, no. 81401243, no. 81572454, and no. 81500394), Shanghai Rising-Star Program (A type) (no. 15QA1400800), The Science Foundation of Shanghai Excellent Youth Scholars (no. 2017YQ042, and The Science Foundation of Shanghai (no. 16411952200, no. 16140902300, no. 14411969800 and no. 17411960600).

\section{Author details}

'Department of Pediatric Surgery, Children's Hospital of Fudan University, and Key Laboratory of Neonatal Disease, Ministry of Health, Shanghai, China. ${ }^{2}$ Medical Scientific Liaison Asian Pacific, Abbott Diagnostics Division, Abbott Laboratories, Shanghai 200032, China. ${ }^{3}$ State Key Laboratory of Oncogenes and Related Genes, Shanghai Cancer Institute, Renji Hospital, Shanghai Jiao Tong University School of Medicine, Shanghai, China

\section{Conflict of interest}

The authors declare that they have no conflict of interest.

\section{Publisher's note}

Springer Nature remains neutral with regard to jurisdictional claims in published maps and institutional affiliations.

Supplementary Information accompanies this paper at (https://doi.org/ 10.1038/s41419-018-0605-y).

Received: 22 December 2017 Revised: 11 April 2018 Accepted: 16 April 2018

Published online: 10 May 2018

\section{References}

1. Hartley, J. L., Davenport, M. \& Kelly, D. A. Biliary atresia. Lancet 374, 1704-1713 (2009).

2. Asai, A., Miethke, A. \& Bezerra, J. A. Pathogenesis of biliary atresia: defining biology to understand clinical phenotypes. Nat. Rev. Gastroenterol. Hepatol. 12, 342 (2015).

3. Bezerra, J. A. et al. Genetic induction of proinflammatory immunity in children with biliary atresia. Lancet 360, 1653-1659 (2002).

4. Feldman, A. G. \& Mack, C. L. Biliary atresia: cellular dynamics and immune dysregulation. Semin. Pediatr. Surg. 21, 192-200 (2012).

5. Hartley, J. L. et al. Investigation of primary cilia in the pathogenesis of biliary atresia. J. Pediatr. Gastroenterol. Nutr. 52, 485-488 (2011).
6. De, C. E., Ivantes, C. A. \& Bezerra, J. A. Extrahepatic biliary atresia: current concepts and future directions. J. De. Pediatr. 83, 105 (2007).

7. Shivakumar, P. et al. Obstruction of extrahepatic bile ducts by lymphocytes is regulated by IFN-gamma in experimental biliary atresia. J. Clin. Invest. 114, 322 (2004).

8. Dong, R., Zhao, R. \& Zheng, S. Changes in epigenetic regulation of CD4+ T lymphocytes in biliary atresia. Pediatr. Res. 70, 555-559 (2011).

9. Penix, L., Weaver, W. M., Pang, Y., Young, H. A. \& Wilson, C. B. Two essential regulatory elements in the human interferon gamma promoter confer activation specific expression in t cells. J. Exp. Med. 178 1483-1496 (1993).

10. Jones, B. \& Chen, J. Inhibition of IFN- $\gamma$ transcription by site-specific methylation during t helper cell development. EMBO. J. 25, 2443-2452 (2006).

11. Yano, S., Ghosh, P., Kusaba, H., Buchholz, M. \& Dan, L. L. Effect of promoter methylation on the regulation of ifn- $\gamma$ gene during in vitro differentiation of human peripheral blood t cells into a th2 population. J. Immunol. 171, 2510 (2003).

12. Matthews, R. P. et al. DNA hypomethylation causes bile duct defects in zebrafish and is a distinguishing feature of infantile biliary atresia. Hepatology $\mathbf{5 3}$, 905-914 (2011).

13. Dong, R., Shen, Z., Zheng, C., Chen, G. \& Zheng, S. Serum microRNA microarray analysis identifies miR-4429 and miR-4689 are potential diagnostic biomarkers for biliary atresia. Sci. Rep. 6, 21084 (2016).

14. Sayed, D. \& Abdellatif, M. MicroRNAs in development and disease. Physiol. Rev. 91, 827-887 (2011).

15. Wang, X. W., Heegaard, N. H. \& Orum, H. Micrornas in liver disease. Gastroenterology 142, 1431-1443 (2012).

16. Lu, C. et al. miR-221 and miR-155 regulate human dendritic cell development, apoptosis, and IL-12 production through targeting of p27kip1, KPC1, and SOCS-1. Blood 117, 4293-4303 (2011).

17. Hand, N. J. et al. MicroRNA profiling identifies miR-29 as a regulator of diseaseassociated pathways in experimental biliary atresia. J. Pediatr. Gastroenterol. Nutr. 54, 186-192 (2012)

18. Morita, S. et al. miR-29 represses the activities of DNA methyltransferases and DNA demethylases. Int. J. Mol. Sci. 14, 14647-14658 (2013).

19. Ogawa, T. et al. Suppression of type I collagen production by microRNA-29b in cultured human stellate cells. Biochem. Biophys. Res. Commun. 391, 316-321 (2010).

20. Sonkoly, E., Stahle, M. \& Pivarcsi, A. MicroRNAs and immunity: novel players in the regulation of normal immune function and inflammation. Semin. Cancer Biol. 18, 131-140 (2008).

21. Pradhan, S., Bacolla, A., Wells, R. D. \& Roberts, R. J. Recombinant human dna (cytosine-5) methyltransferase. i. expression, purification, and comparison of de novo and maintenance methylation. J. Biol. Chem. 274, 33002 (1999).

22. Okano, M., Bell, D. W., Haber, D. A. \& Li, E. DNA methyltransferases dnmt3a and dnmt3b are essential for de novo methylation and mammalian development. Cell 99, 247-257 (1999).

23. Hamilton, J. P. Epigenetics: principles and practice. Dig. Dis. 29, 130-135 (2011).

24. Jones, P. A. Functions of DNA methylation: islands, start sites, gene bodies and beyond. Nat. Rev. Genet. 13, 484-492 (2012).

25. Pogribny, I. P. \& Beland, F. A. DNA hypomethylation in the origin and pathogenesis of human diseases. Cell. Mol. Life. Sci. 66, 2249-2261 (2009).

26. Portela, A. \& Esteller, M. Epigenetic modifications and human disease. Nat. Biotechnol. 28, 1057-1068 (2010)

27. Balada, E., Ordi-Ros, J. \& Vilardell-Tarres, M. DNA methylation and systemic lupus erythematosus. Ann. N. Y. Acad. Sci. 1108, 127-136 (2007).

28. Weisenberger, D. J. et al. Analysis of repetitive element DNA methylation by MethyLight. Nucl. Acids Res. 33, 6823-6836 (2005).

29. Eads, C. A. et al. MethyLight: a high-throughput assay to measure DNA methylation[J]. Nucl. Acids Res. 28, E32 (2000).

30. Egger, G., Liang, G., Aparicio, A. \& Jones, P. A. Epigenetics in human disease and prospects for epigenetic therapy. Nature 429, 457 (2004).

31. Lalvani, A. \& Pareek, M. Interferon gamma release assays: principles and practice. Enferm. Infecc. Microbiol. Clin. 28, 245-252 (2010).

32. Zhao, R. et al. MicroRNA-155 modulates bile duct inflammation via targeting suppressor of cytokine signaling 1 in biliary atresia. Pediatr. Res. 82, 1007-1016 (2017).

33. Udomsinprasert, W. et al. Global methylation, oxidative stress, and relative telomere length in biliary atresia patients. Sci. Rep. 6, 26969 (2016). 